\title{
Time resolved $3 D$ interferometric imaging of a section of a negative leader with LOFAR
}

\author{
O. Scholten $\odot,{ }^{1, *}$ B. M. Hare $\odot,{ }^{2}$ J. Dwyer, ${ }^{3}$ N. Liu $\odot,{ }^{3}$ C. Sterpka $\odot,{ }^{3}$ S. Buitink, ${ }^{4,5}$ T. Huege, ${ }^{6,5}$ \\ A. Nelles, ${ }^{7,8}$ and S. ter Veen ${ }^{9}$ \\ ${ }^{1}$ University Groningen, Kapteyn Astronomical Institute, Landleven 12, \\ 9747 AD Groningen, The Netherlands, \\ University of Groningen, KVI Center for Advanced Radiation Technology, Groningen, The Netherlands, \\ and Interuniversity Institute for High-Energy, Vrije Universiteit Brussel, Pleinlaan 2, \\ 1050 Brussels, Belgium \\ ${ }^{2}$ University Groningen, Kapteyn Astronomical Institute, Landleven 12, \\ 9747 AD Groningen, The Netherlands \\ ${ }^{3}$ Department of Physics and Space Science Center (EOS), University of New Hampshire, \\ Durham, New Hampshire 03824, USA \\ ${ }^{4}$ Department of Astrophysics/IMAPP, Radboud University Nijmegen, 6525 AJ Nijmegen, The Netherlands \\ ${ }^{5}$ Astrophysical Institute, Vrije Universiteit Brussel, Pleinlaan 2, 1050 Brussels, Belgium \\ ${ }^{6}$ Institut for Astroparticle Physics, Karlsruhe Institute of Technology (KIT), \\ P.O. Box 3640, 76021 Karlsruhe, Germany \\ ${ }^{7}$ Erlangen Center for Astroparticle Physics, Friedrich-Alexander-Univeristät Erlangen-Nürnberg, \\ D-91058 Erlangen, Germany \\ ${ }^{8}$ DESY, Platanenallee 6, 15738 Zeuthen, Germany \\ ${ }^{9}$ Netherlands Institute for Radio Astronomy (ASTRON), 7991 PD Dwingeloo, The Netherlands
}

(Received 24 April 2021; accepted 19 July 2021; published 15 September 2021)

\begin{abstract}
We have developed a three dimensional interferometric beamforming technique for imaging lightning flashes using very-high frequency (VHF) radio data recorded from several hundred antennas with baselines up to $100 \mathrm{~km}$ as offered by the Low Frequency Array. The long baselines allow us to distinguish fine structures on the scale of meters, while the large number of antennas allow us to observe processes that radiate at the same intensity as the background when using a time resolution that is close to the impulseresponse time of the system, $100 \mathrm{~ns}$. The new beamforming imaging technique is complementary to our existing impulsive imaging technique. We apply this new tool to the imaging of four stepped negative leaders in two flashes. For one flash, we observe the dynamics of corona bursts that are emitted in the stepping process. Additionally, we show that the intensity emitted in VHF during the stepping process follows a power law over 4 orders of magnitude in intensity for four leaders in two different lightning storms.
\end{abstract}

DOI: $10.1103 /$ PhysRevD.104.063022

\section{INTRODUCTION}

Due to the fact that it is unpredictable, violent, and often hidden in clouds, the common phenomenon of lightning still harbors many secrets, such as the conditions for initiation and the processes that allow the formation of conducting ionized channels called leaders. During any part of its development, a lightning flash emits copious amounts of electromagnetic radiation over a very broad frequency spectrum, ranging from static electric fields to gamma-ray emission. For this work, we use the emission in the veryhigh frequency (VHF) band to image the three dimensional

* Corresponding author. O.Scholten@rug.nl
(3D) lightning development in time. The major advantage of VHF-imaging over, for example, optical imaging is that the VHF is not obscured by the presence of clouds, allowing for large distance observations.

The imaging of lightning in three dimensions using VHF-interferometric-based methods has a history dating back to the early work of [1], where 3D is reached by combining angular measurements from two different stations. This work was followed up by others, such as [2-4], and culminated recently in the work of [5] reaching a resolution of $200 \mathrm{~m}$ using six antennas.

We have developed similar techniques for imaging lightning with Low Frequency Array (LOFAR) [6], which has resulted in much improved resolution. In this previous work we had used the signals of several hundred antennas 
for 3D imaging [7]. In this impulsive-imaging approach the time of the peak in the cross correlation between a pulse on the reference station and pulses in other stations is used in a chi-square fitting procedure to find the most likely location for the source. This impulsive imager was optimized a step further in [8] and can find over 200 source locations per millisecond, where meter-scale accuracy is reached.

Despite the history in the lightning field of naming these previous techniques "interferometry," few of them $[9,10]$ use actually true interferometry, in the sense of coherently summing the electric fields measured by many antennas. In this work we show the results of using a true interferometric beamforming technique for imaging lightning in 3D using hundreds of LOFAR antennas with maximal baselines of $100 \mathrm{~km}$, using the analytic (complex-valued) signal. In beamforming the signals from hundreds of antennas are coherently added, and the imaged volume is rastered into voxels, volumetric pixels. Where most interferometric approaches integrate over considerable time periods, we divide the beamformed time trace for each voxel in narrow slices. A slice may be as small as $100 \mathrm{ns,}$, about twice the impulse-response time of the LOFAR antennas. For each time slice and each voxel, the coherent intensity is calculated, thus forming a 4D (three space and one time dimension) intensity image. For each time slice, the position of maximal coherent intensity is taken as the position of the source. In spite of the high time resolution we still resolve sources that emit with intensity well below the noise limit, where the noise level of a single antenna is dominated by the galactic background [11] (after removing narrow-band radio-frequency interference). Since we can reach a time resolution of the order of the impulse-response time of our system, we name this procedure time resolved interferometric 3-dimensional (TRI-D) imaging. In Sec. II the TRI-D imaging technique is discussed in detail, and a first application to negative leaders is given in Sec. III.

Lightning, being an electric discharge phenomenon, has positively and negatively charged propagating structures, called leaders. While the positive leaders propagate in a rather continuous way, the negative leaders, in contrast, propagate discontinuously through a stepping process. In this stepping process copious amounts of VHF are emitted, making them ideal test cases for our imaging method. As a first application, we thus have used TRI-D imaging to investigate negative stepped leaders in Sec. III. The main drawback of interferometric 3D imaging is that it is compute intensive, thus without large dedicated compute resources only a relatively small region of space can be imaged. In Sec. III A we explain our two-step approach, where the entire lightning flash is imaged with the impulsive imager [8], and from this image the interesting parts are selected that are subsequently imaged using the TRI-D imager. We show in Sec. III B that the TRI-D imager allows to resolve the dynamics of a corona burst [12-15] for some negative leaders. As a second application, we show in Sec. IIIC that the intensity spectrum of
VHF-pulses emitted from negative leaders follows an almost perfect inverse-square power law over 4 orders of magnitude (with a scaling exponent of -1.8). We end with a short discussion in Sec. IV.

\section{METHODS}

The LOFAR infrastructure consists of several thousand antennas distributed in stations over much of Europe, with a dense core in the Netherlands. For the present analysis, we use the antennas installed in the Netherlands. The LOFAR antennas are arranged in stations each containing 96 low band antennas, inverted v-shaped dipoles operating in the $30-80 \mathrm{MHz}$ band, and a similar number of high band antennas (100-200 MHz, not used here). For instrumental reasons, the data of 12 dipoles each from all 37 Dutch stations is used. The stations have a roughly logarithmic spacing, and the geographic distribution is shown in Fig. 1. The maximal baseline is $100 \mathrm{~km}$. At the core of LOFAR, called the Superterp, the antenna stations are more closely packed. Upon a trigger, the random access memory (RAM) buffers containing raw data from every antenna are frozen and read out over glass fiber to a central storage. This allows for off-line processing of the time traces (sampled at $200 \mathrm{MHz}$ ) of all antennas. The atomic clocks in the stations allow for a timing stability of a fraction of a

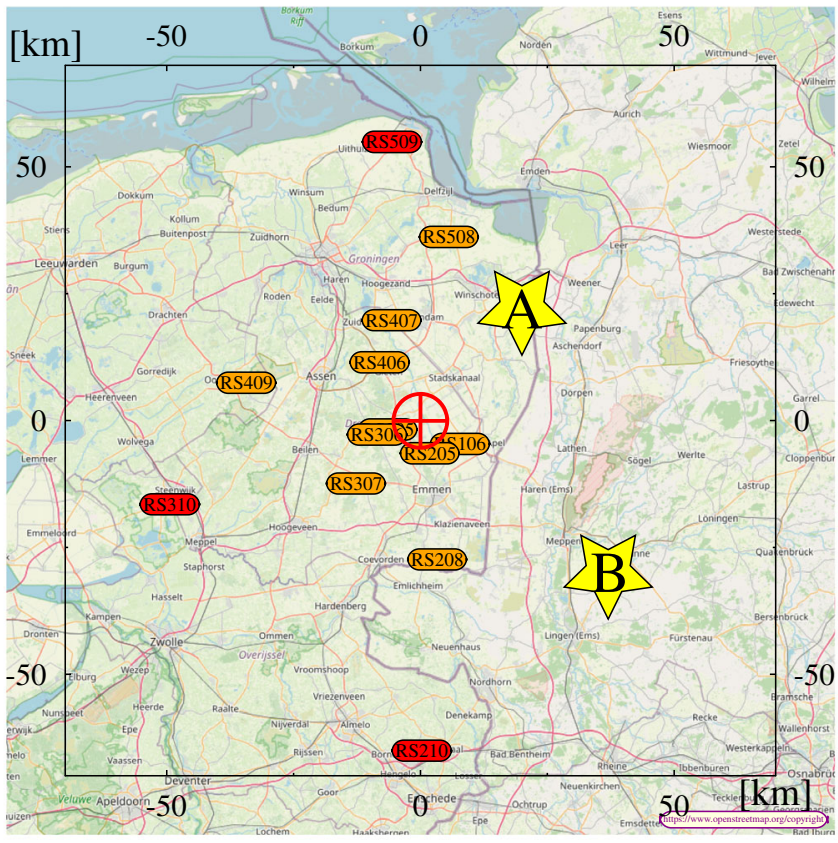

FIG. 1. Layout of the Dutch LOFAR stations. The core of LOFAR is indicated by the red $\bigoplus$ sign, while the yellow stars show the general location of flashes A and B that are discussed in this work. The black frame indicates the general area $\left(140 \times 140 \mathrm{~km}^{2}\right)$ where flashes can be mapped accurately and is centered at the LOFAR core. Stations marked in red are not included in TRI-D imaging (see text). 

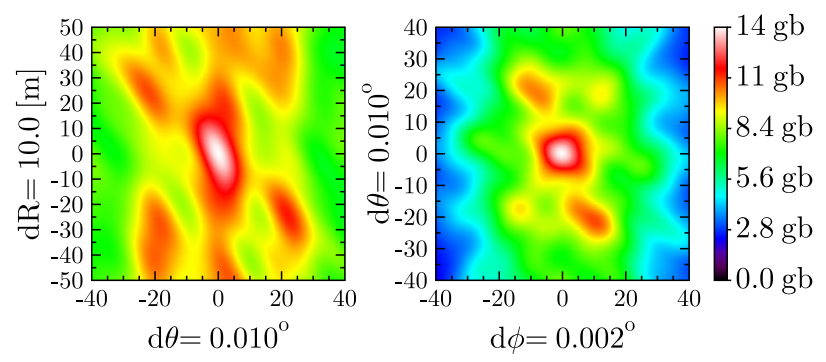

FIG. 2. A typical interferometer image for flash B (for an integration time of $5 \mu \mathrm{s}$ ). The axes show the grid in voxel number where $(0,0,0)$ is the center voxel in the image cube. Both images are made for a thin (one voxel thick) section through the center voxel. To show the general features of the intensity plot, the hypercube is chosen such that the maximum intensity is at the center voxel, which generally is not the case. The typical length scale for $d \phi$ and $d \theta_{e}$ is about $1 \mathrm{~m}$. The intensity unit $(\mathrm{gb})$ is explained in Sec. II C.

nanosecond over the recording time span of maximally five seconds.

The basic implementation of beamforming imaging is relatively straightforward. The part of the atmosphere where the lightning is to be imaged is rastered into voxels, see Sec. II A. For each voxel the time traces of all antennas are summed, while accounting for the difference in travel time to each antenna. This yields for each voxel a time trace, which is the coherent sum of all antennas, as discussed in Sec. II A. Adding the traces for the different voxels is always performed for a fixed time span in one particular "reference" antenna, usually an antenna from the core of LOFAR. Thus, we can find the source location of a particular structure seen in the trace of the reference antenna. The choice of reference antenna is not important as long as it is in the dense core.

The time trace of each voxel is cut in slices of a fixed duration. The slicing is done such that they are synchronous when correcting for the signal travel time from the reference antenna to the voxel. For all slices, the coherent intensity is determined resulting in a voxelated intensity profile for each time slice, similar to what is shown in Fig. 2. For each time slice, the maximum of this voxelated intensity profile is determined and used as the source location, as discussed in more detail in Sec. II D. Plotting the position of all sources will result in the beam-formed (or interferometric) image of a segment of the flash in space and time, where the length of the time slices determines our final time resolution since any detail within a slice is summed over. In the following, the different steps involved in this procedure are discussed in more detail.

\section{A. Space and time grids and summing time traces}

The part of the atmosphere that is of interest is rastered into voxels. We have found that this is most conveniently done in spherical coordinates with the center at the reference antenna in the dense LOFAR core. In this way we can more easily account for the fact that our resolution in the two transverse directions is much better than in the radial direction. The optimal grid spacing is dependent on the areal density of the antenna locations. For the example discussed in this work, we include antennas within a $50 \mathrm{~km}$ distance from the core, where the antennas are distributed on an irregular logarithmic grid, with a dense core that is designed to be optimal for astrophysical applications, see [6] and Fig. 1. The voxel grid is chosen such that the maximal time shift for any antenna for two bordering voxels is about $1 \mathrm{~ns}$; our time calibration accuracy. This implies, for the examples discussed in Sec. III, a grid spacing of $0.003^{\circ}$ in the azimuth angle $(\hat{\phi}), 0.01^{\circ}$ in elevation angle $\left(\hat{\theta}_{e}\right.$, upwards from the horizon), and $10 \mathrm{~m}$ in the radial direction $(\hat{R})$. For a source at $50 \mathrm{~km}$ distance, this implies a grid spacing of about $1 \mathrm{~m}$ transverse to the line of sight (from the reference antenna) and $10 \mathrm{~m}$ along the line of sight. A typical grid used in Sec. III spans over $(60 \times$ $30 \times 20)$ voxels in $\left(\hat{\phi}, \hat{\theta}_{e}, \hat{R}\right)$ and will be referred to as the image cube.

A section of the time trace in the reference antenna is selected with typically a length of $0.3 \mathrm{~ms}$. For each voxel, the time traces of all antennas are added after time shifting them to account for the travel time differences of a signal from the voxel. This yields the coherent (or beam formed) time trace for that voxel. All coherent voxel time traces are evaluated at identical times for the reference antenna.

The actual time shift of the time traces is done in Fourier space by applying a frequency-dependent phase shift to the trace for each antenna. After summation, the traces are transformed back to the time domain. The actual antenna time traces are taken longer than the required $0.3 \mathrm{~ms}$ to be able to account for the maximal relative time shift over the full voxelated volume. We thus obtain for each voxel a coherent time trace, with all antennas contributing, of $0.3 \mathrm{~ms}$ length with additional trailing ends where there will be partial coherence. These trailing ends are excluded from the analysis.

The intensity may be integrated over the full time trace of $0.3 \mathrm{~ms}$, which washes out all time dependence over the integration range. However, a much better time resolution is generally required in order to image the dynamics of the flash. To obtain this, we cut the time trace from each voxel into narrow slices, and we integrate the power over each slice for every voxel to obtain the voxelated intensity profile for each slice, giving an image like Fig. 2. The optimal slice length depends on the research question of interest, taking it short resolves finer time structures but makes the imaging more noisy, taking it long improves the signal-to-noise ratio, and thus the sensitivity, but increases the chance that multiple sources appear simultaneously and are mixed up. The smallest time step, where subsequent time-frames are considered to be independent, is the width of the impulse-response function. The impulse-response 
time is determined by the frequency dependence of the LOFAR antennas, amplifiers, and filters. An easy estimate of this is obtained from the full width at half maximum of the narrow peaks in the spectrum, about $50 \mathrm{~ns}$. As shown in Sec. III very stable results are obtained even for a time slice as short as $100 \mathrm{~ns}$, which is a little longer than the impulseresponse time. Since we have shown that we can reach down close to the impulse-response time of the system, we name this TRI-D imaging.

\section{B. Antenna weighting}

Since the array of antennas has an extent that is comparable to the distance to the source region, the received signal strength varies greatly over the array. If this is not taken into account in the analysis, then one would effectively use the antennas that are near the source region only and thus potentially use only a fraction of the imaging accuracy of the array as a whole. To improve this aspect of the performance, we include weighting factors for the antennas to compensate for the imbalance in signal strength. In interferometry applications for astronomy (2D, not 3D imaging) there is a considerable amount of literature regarding the advantages and disadvantages of different weighting schemes, see $[16,17]$ for comprehensive reviews.

For this work, we have chosen a weighting scheme that compensates in lowest order the signal strength variations in the antennas, due to their distance from the source, where the weight is capped for antennas far from the source for which the signal to noise ratio becomes unfavorable. In addition it takes into account the fact that the largest density of antennas is at the core.

The amplitude of an emitted signal drops inversely proportional to distance $R_{a s}=\sqrt{D_{a s}^{2}+h_{s}^{2}}$, where $D_{a s}$ is the distance from a certain antenna to the source in the horizontal plane, and $h_{s}$ denotes the altitude of the source. For this weighting scheme, it is assumed that the antennas are all in a horizontal plane since that part of the Netherlands is flat to a good approximation, and in all other interferometry calculations, the proper 3-dimensional locations of the antennas are used. Another important factor to account for is the antenna gain that depends on the azimuth $\phi$ and elevation $\theta_{e}$ angles of the source with respect to the antenna. The antenna gain vanishes for sources at the horizon and thus (since it is an analytic function of the angles) depends on $\theta_{e}$ as $\sin \left(\theta_{e}\right)$. For almost all cases of interest, the sources are at small elevation angles for which we can assume $\sin \left(\theta_{e}\right) \approx h_{s} / R_{a s}$, where the antenna position enters in $R_{a s}$ only.

The antenna gain depends also on $\phi$, as well as on the polarization of the signal. Since an analysis of the polarization of the radiation falls outside the scope of this work, as it would make the analysis considerably more complicated, we have ignored this dependence. Even though the antennas are not in a small azimuthal angle range from the source (full opening angle about $60^{\circ}$, see Fig. 1), we still achieve high-quality results.

Combining the $1 / R_{a s}$ drop of signal strength with the proportionality of the antenna gain to $\sin \left(\theta_{e}\right)$, we obtain an antenna weighting factor

$$
W_{a}= \begin{cases}R_{a s}^{2} / R_{r s}^{2} & \text { if } R_{a s}^{2} / R_{r s}^{2}<W_{\max } \\ W_{\max } & \text { if } R_{a s}^{2} / R_{r s}^{2} \geq W_{\max }\end{cases}
$$

where the weight is normalized to unity for the reference antenna at a distance of $R_{r s}$ to the source. In addition, the weight is capped to a maximum of $W_{\max }=1.2$ for distant stations, where the signal-to-noise ratio is getting worse. It is conceivable that a different choice for $W_{\max }$, for example by making it dependent on the signal-to-noise ratio, will improve the image accuracy. The weighting factor thus compensates in leading order for the zenith angle dependence of the antenna function, as well as the radial falloff of the signal strength, but does not account for multipath propagation near the ground (reflections off the ground [18] or from objects in the vicinity of the antenna) as this is very difficult to simulate. Multipath propagation will adversely affect the resolution.

\section{Antenna calibration and intensity}

The antenna timings are calibrated for each flash following the procedure outlined in $[7,8]$. Per flash, 20-30 bright stand-alone pulses are selected from the whole flash, where care is taken that their source locations roughly cover the extent of the flash. For all sources, the location, as well as the antenna timings, are searched in a simultaneous fit using the search algorithm discussed in [8].

Since for interferometry the stability of the phase of the signal over all antennas is important, we perform an additional check of this phase for a single distinct pulse in the spectrum selected for TRI-D imaging. If this phase is off by more than $90^{\circ}$, then the antenna will not be used. Usually this eliminates less than $1 \%$ of all antennas. This may also result in eliminating a whole station from the analysis for a particular flash. The latter could be due to the fact that we have ignored the azimuthal angle dependence of the antenna function.

The gain of each antenna is calibrated by normalizing the noise level to unity. The noise level is determined from the parts of the recorded trace for which there is no lightning activity detected within $0.3 \mathrm{~ms}$. Since this noise level is largely due to nonterrestrial sources, i.e., radiation produced by the Milky Way, we name this the galactic background (gb) level.

The coherent, or interferometric, intensity is calculated as the intensity of the signal from the source as received by the reference antenna and is expressed in units of gb. It is tacitly assumed that the azimuth-angle dependence of the 

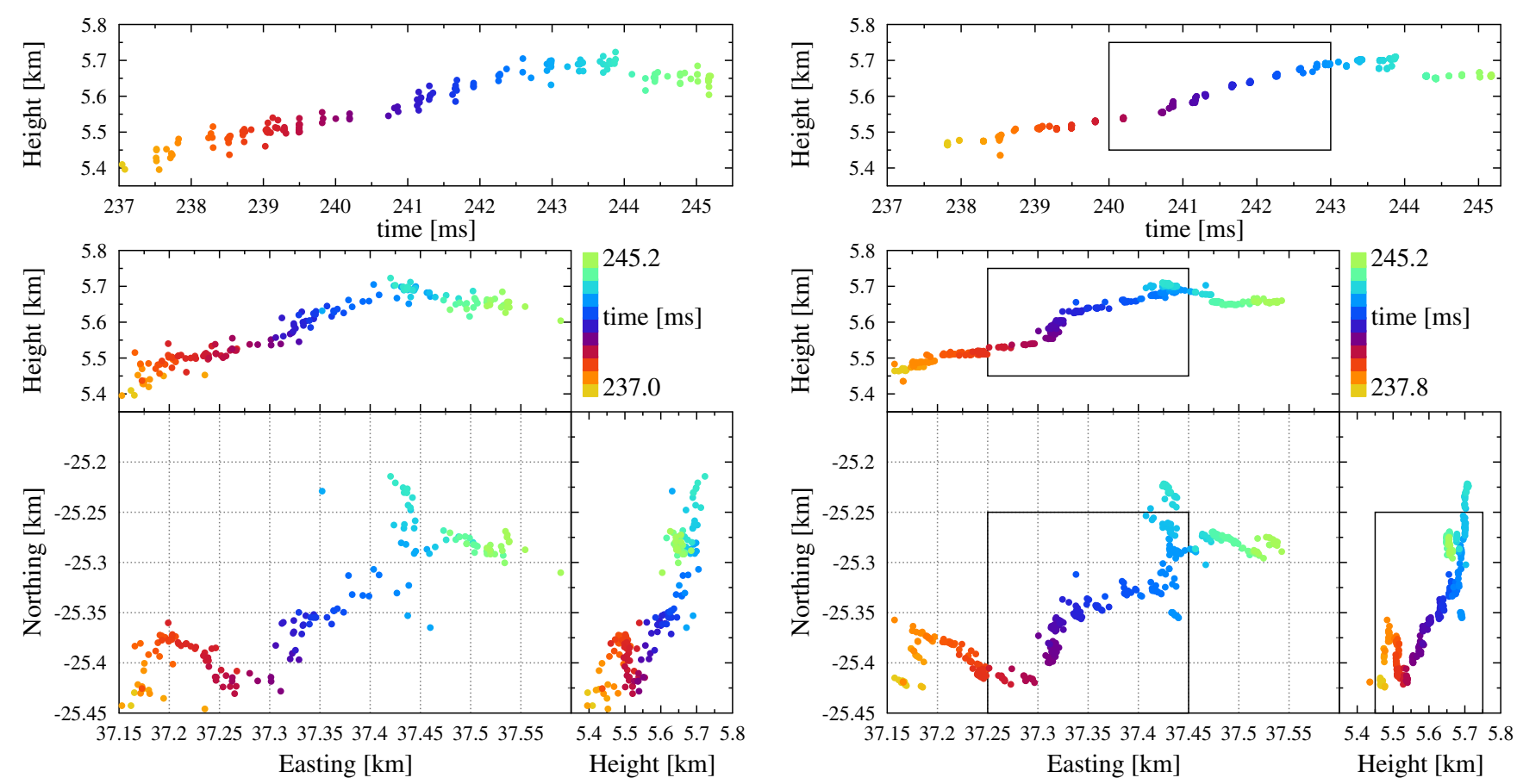

(a) impulsive imager

(b) TRI-D imager

FIG. 3. A comparison of the images for section "NL-B1" from a negative leader of flash B (see Table I) as obtained by the impulsive imager [panels (a)] and the TRI-D imager [panels (b)] using the X dipoles from LOFAR. The boxes on the right indicate the enlarged volume for Fig. 4(a).

antenna gains is limited and is effectively averaged out. The elevation-angle dependence is to a large extent accounted for by the weighting factors (see Sec. II B) when calculating the coherent intensity. A very rough estimate of the emitted pulse power can be obtained by following the arguments presented in [19], the supplemental material, that follows the same arguments as presented in [20]. The received power of galactic background in the $30-80 \mathrm{MHz}$ frequency window is approximately $P_{g b}=210^{-12} \mathrm{~A} \mathrm{~W}$, where $A \approx$ $1 \mathrm{~m}^{2}$ is the effective area of a LOFAR antenna. The power of a pulse that is recorded at $P_{s} \mathrm{gb}$ in a $100 \mathrm{~ns}$ window is thus $P_{s} \times 210^{-12} \mathrm{~A} \times 10^{-7} \times R_{r s} / h[\mathrm{~J}]$, where the last factor accounts for the projected area of the antenna, which is a crude approximation to the antenna function as discussed in Sec. II B. The emitted energy of the source, assuming dipole emission, can now be estimated as $E_{s}=$ $P_{s} R_{r s} / h \times 210^{-19} \times 4 \pi R_{r s}^{2} / 2=4 \pi P_{s} R_{r s}^{3} / h \times 10^{-19}[\mathrm{~J}]$. A typical source in the leader shown in Fig. 3 at $\left(R_{r s}=45 \mathrm{~km}, \mathrm{~h}=5.5 \mathrm{~km}\right)$ thus emits approximately an energy $E_{s}=P_{s} 10^{-11} \mathrm{~J}$ in a short pulse.

All LOFAR antennas used in this work are inverted v-shape dipoles, where $\mathrm{X}(\mathrm{Y})$ dipoles, corresponding to odd (even) numbered antennas, are oriented in the NE-SW (NW-SE) plane. Our analysis is performed separately for $\mathrm{X}$ and $\mathrm{Y}$ dipoles since they differ significantly in their sensitivity to polarized radiation. The antenna function (the Jones matrix, specifying for each dipole and polarization the gain depending on angle and frequency) has to be used [6] to convert the measured intensity to the absolute intensity of the source, which we have not done in this work. The absolute calibration of the LOFAR antennas is performed in [11]. Extracting intensities (and even polarization) of a source is considerably simplified if it is assumed that the source is pointlike, emitting a delta pulse (flat frequency spectrum over our frequency range), and linearly polarized, however, such an analysis falls outside the scope of the present work.

\section{Determining the location of maximal intensity}

Figure 2 shows a typical intensity profile for a time slice of $10 \mu \mathrm{s}$. For this particular one, the coordinates of the image cube have been chosen such that the peak intensity is at the center. Generally this is, of course, not the case. The procedure used to determine the position of the maximum is discussed in this section. Besides the main maximum there are several secondary maxima, due to so-called side beams, a well-known feature in interferometric imaging. The positions and intensities of these side beams are determined by the antenna configuration. The LOFAR irregular lattice of antennas suppresses the intensities of these side beams to a large extent. Secondary maxima could also be due to background noise or due to multiple sources in the same time slice. It is straightforward to determine the voxel with maximal intensity. To reach an intervoxel 
accuracy, we have implemented two interpolation procedures, quadratic and barycentric interpolation.

(i) Quadratic: The intensity of the voxels around the voxel with the maximum intensity are fit with a paraboloid,

$I_{p}(\vec{x})=I_{0}+\sum_{i}\left[\frac{1}{2} A_{i} x_{i}^{2}+B_{i} x_{i}\right]+\sum_{i<j} R_{i, j} x_{i} x_{j}$,

where $x_{i}$ is the $i^{\text {th }}$ grid coordinate, $i=1,2,3$. The coefficients $I_{0}, A_{i}$, and $R_{i, j}$ are fitted to the grid points bordering the maximum. The intervoxel maximum $\overrightarrow{x_{p}}$ is taken at the point where the paraboloid reaches its maximum.

(ii) Barycentric: The barycentric maximum is calculated as

$$
\vec{x}_{b}=\sum_{\vec{x}}\left[\left(I(\vec{x})-I_{\mathrm{Th}}\right) \vec{x}\right] / \sum_{\vec{x}}\left[\left(I(\vec{x})-I_{\mathrm{Th}}\right)\right],
$$

where the sum runs over all voxels with an intensity exceeding the threshold value $I_{\mathrm{Th}}$. This threshold is taken as $I_{0} / 1.2$ (where $I_{0}$ is the maximum voxel intensity) or the largest value of a voxel on the outer surface of the grid, whichever is larger.

When there are many active sources in small area, the barycentric interpolation will yield some weighted average position, while the quadratic interpolation yields the position of the strongest source. It is observed that the details of intensity surface are complicated with small ripples (order $10 \%$ in intensity over distances of $20 \mathrm{~m}$ depending on the source location) likely due to side beams. These ripples make the quadratic interpolation unstable for a coarse grid. Using barycentric interpolation, these ripples are efficiently averaged yielding a properly interpolated maximum. The examples shown in Sec. III use a fine grid, and for this reason the quadratic interpolation is used.

\section{NEGATIVE LEADERS}

As an example of the possibilities offered by the new TRI-D imager we apply it to some normal negative leaders. These are selected as their structure is assumed to be relatively simple, and one thus obtains a good insight into the intrinsic accuracy of the TRI-D imager. We use two negative leaders from each of two flashes, flash A occurring on April 24, 2019 at 21:30:56.221 UTC and flash B on August 14, 2020 at 14:14:58.669 UTC. These times are taken as $t=0$ for each flash. We show an enlarged view of the fine structure of one negative leader of flash B and statistics for all four negative leaders.

The location of the two flashes with respect to the LOFAR core is shown in Fig. 1. Both flashes have been imaged using the impulsive imager, where flash A was
TABLE I. The negative leader sections that are used for further analysis.

\begin{tabular}{lrrclc}
\hline \hline Flash & Leader & Time $(\mathrm{ms})$ & North $(\mathrm{km})$ & East $(\mathrm{km})$ & Height $(\mathrm{km})$ \\
\hline $\mathrm{A}$ & NL-A1 & $170-178$ & $21-23$ & $18-20$ & $2.5-3.5$ \\
$\mathrm{~A}$ & NL-A2 & $177-182$ & $21-23$ & $21-22$ & $3 .-3.5$ \\
$\mathrm{~B}$ & NL-B1 & $238-245$ & $-25--25$ & $37-38$ & $5.3-5.8$ \\
$\mathrm{~B}$ & NL-B2 & $540-552$ & $-34--34$ & $36-37$ & $4.9-5.5$ \\
\hline \hline
\end{tabular}

reported on in $[8,21]$ and flash $\mathrm{B}$ in [22]. For the impulsive imager all stations are used, including those marked in red in Fig. 1, while for TRI-D imaging we have excluded these stations because they are at a distance larger than $50 \mathrm{~km}$ from the LOFAR core. This limit on distance is taken since for further distances the signal becomes weak, or the spread in angles to the source too large. For flash B, also station RS208, RS508, and RS307 were excluded since for these the phase stability for selected pulses could not be shown, see Sec. II C.

The sections of the negative leaders, which we will use for the discussion in this work, are given in Table I. In Sec. III A we show that, while the image quality on a large scale may be somewhat better for the TRI-D imager as compared to the impulsive imager, this does not outweigh the additional effort in CPU power. As is shown in Sec. III B, the true strength of the TRI-D imager lies in resolving fainter sources, while keeping good time resolution. Another strong point of the TRI-D imager is that it is complete in the sense that it will locate all sources with a strength exceeding the background in the image-cube with only minor caveats. The impulsive imager is, in this respect, much less complete since the located sources are not restricted by the small image cube (which can also be an advantage) and, furthermore, need to be well separated from earlier and later sources. The completeness of the TRI-D-imaged sources allows to analyze the VHF-intensity spectrum, as discussed in Sec. III C.

\section{A. Larger scale structures}

Interferometric imaging is rather expensive. For the present implementation, it typically takes half a day on a single CPU to produce an TRI-D image of a volume of about $1 \mathrm{~km}^{3}$ for a trace of $0.3 \mathrm{~ms}$, while it takes about one hour to image a section of $100 \mathrm{~ms}$ of a flash with the impulsive imager, however, since the interferometric imaging code can easily be paralleled, this problem may not be as constraining at it appears. Both estimates are for a typical LOFAR configuration with a few hundred antennas. In Fig. 3 the results of the two imaging procedures are compared for a section of about $1 \mathrm{~km}$ of a negative leader from flash B. This represents only a small section of the full flash that covers an area of the order $25 \times 10 \mathrm{~km}^{2}$ and is active for $2 \mathrm{~s}$. For the TRI-D image [Fig. 3(b)], only strong sources $(I>30 \mathrm{gb})$ are plotted resulting in 399 sources, 

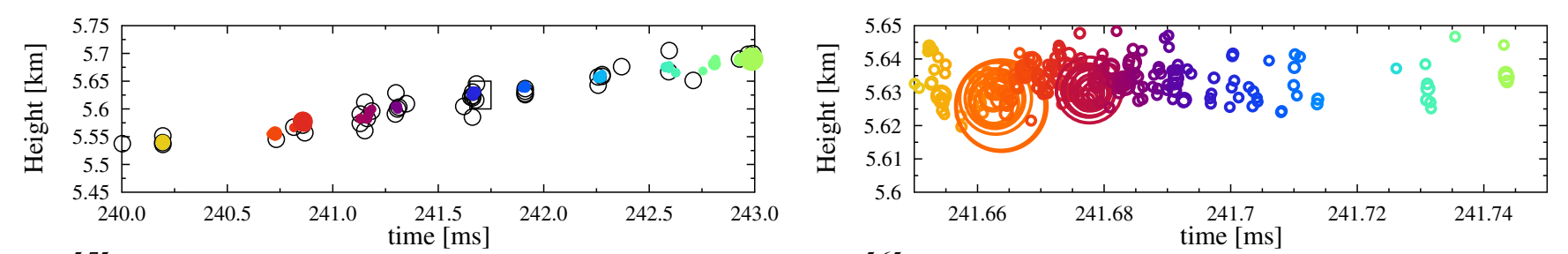

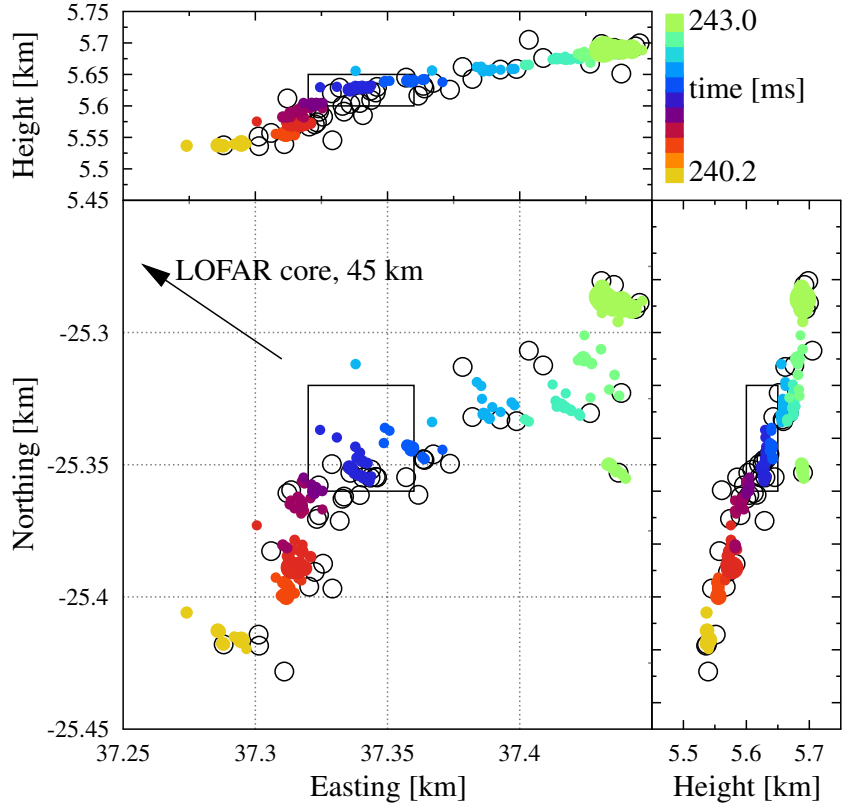

(a) Zoom in on Fig. 3

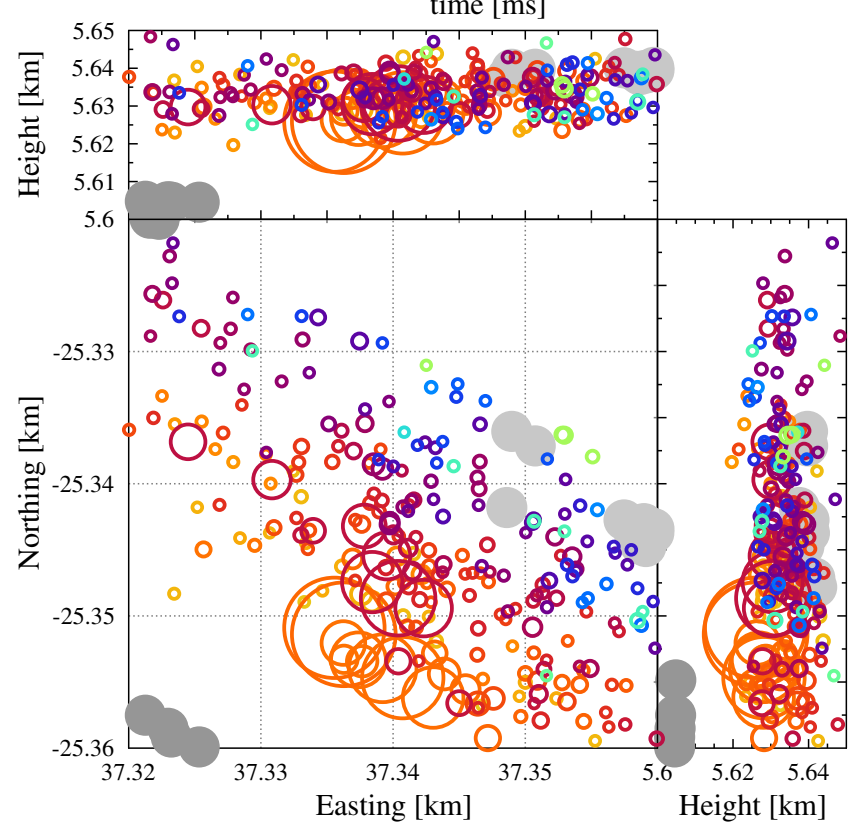

(b) Zoom in on Fig. 4a

FIG. 4. Side a shows an enlargement on a section of the negative leader shown in Fig. 3. The sources found by the impulsive imager (44 sources) are indicated by black open circles and in color those from TRI-D with a $I>30$ gb (99 sources). The arrow indicates the radial direction and points to the core of LOFAR. Side (b) shows an even further enlargement on one particular burst, corresponding to the rectangular boxes in (a), as imaged using TRI-D with an intensity threshold of $I>0.3$ gb showing 153 sources. In light (darker) grey the more intense TRI-D sources are shown that fall after (before) the time frame of the plot. The area of the circles is proportional to the intensity for the colored strong sources.

while the quality cuts on the impulsive image [Fig. 3(a)] leaves 159 sources. The two imaging methods are subject to different systematic and statistical uncertainties and have a different resolution. One should, therefore, focus on general structures and not on individual points and as is seen from Fig. 3, there is no significant difference between the two. The sources in the TRI-D image show in the plane view a bit less scatter than those from the impulsive imager, while in the vertical direction the scatter is reduced by at least a factor 2. Future work will have to show if this improvement is due to the particular location of this leader, with respect to the LOFAR stations, or if it is more general.

We observe that the received coherent power integrated over $0.3 \mathrm{~ms}$ for a section of a negative leader of flash $\mathrm{A}$, located at the NE of the array, is about twice as large for the $\mathrm{Y}$ dipoles as it is for the X dipoles. For flash B (at the SE of the core) the intensity ratio is reversed. This is due to the azimuth-angle dependence of the antenna gain. For sources in the NE, horizontally polarized radiation is recorded almost exclusively in the $\mathrm{Y}$ dipoles with a gain (in power) that is about a factor 2 larger than that for vertical polarization recorded exclusively by the $\mathrm{X}$ dipoles in this configuration, while for sources in the SE the ratio is reversed. The observed asymmetry in the response of the two dipoles is thus not an indication of a strong polarization of the emitted VHF radiation.

\section{B. Detailed view of a negative leader}

The strength of TRI-D imaging is shown in Fig. 4, where enlargements are made at two different levels on the leader track shown in Fig. 3. Figure 4(a) shows in color the stronger TRI-D sources $(I>30 \mathrm{gb})$ only, while for the enlarged Fig. 4(b) all TRI-D sources with an intensity $I>$ $0.3 \mathrm{gb}$ (i.e., with a strength below the noise level of a single antenna) are shown.

One aspect evident from Fig. 4 is that our imaging accuracy is very directional dependent, as was remarked already in Sec. II A. Sources occurring in a narrow time window, i.e., have the same color, show a much larger spread in the $(\hat{R})$ direction [radially outward from the core at $(0,0)$, indicated by the black arrow on the plane view of 
Fig. 4(a)] than in the transverse direction, about a factor 10 difference.

Figure 4 shows clearly that the real strength of TRI-D imaging lies in its ability to image the finer details of a flash. The time-colored sources in Fig. 4(a) are obtained through TRI-D imaging, while the sources marked by black circles have been found using the impulsive imager, using the same quality and interferometric-intensity cuts as in Fig. 3 for both. The top panel clearly shows that also this leader has a distinct burst structure that is typical for negative leader propagation and was analyzed extensively in [19]. Figure 4(b) shows an enlargement on the burst indicated by the black boxes in Fig. 4(a). The size of the colored circles reflects source intensity. The limit on the intensity has been decreased to $0.3 \mathrm{gb}$, well below the noise level in the reference antenna, which is the reason that many more sources show (a total of 254 for the enlarged section). As is to be expected, the positioning accuracy of the weaker sources is less, and they thus show more scatter, in particular, in the radial direction. The grey sources in the right panels mark the positions of intense TRI-D-imaged sources that fall outside the imaged time span of 241.65$241.75 \mathrm{~ms}$ used for the colored sources. The earlier sources are darker grey (at time $t<241.31 \mathrm{~ms}$ ) and the lighter ones occur later than the colored sources (at $t>241.89 \mathrm{~ms}$ ). For all grey sources, the dot size is constant.

In the plane view of Fig. 4(b) the leader propagates from the lower left corner to the middle right, i.e., from the dark grey dots to the light grey ones. The height vs time panel, the top of Fig. 4(b), shows that the burst itself has a very distinct structure, starting with some intense sources, the corona burst itself, followed by weaker sources with a decreasing density. From the plane view, the bottom panel, it is seen that they follow the propagation direction of the leader, as expected for streamers ensuing from a corona burst. One may even distinguish a double burst, one starting with sources in orange at $t=241.662 \mathrm{~ms}$ and one in burgundy maroon starting $0.014 \mathrm{~ms}$ later. As discussed in the following paragraph, the spread of the sources in the radial direction is probably showing the imaging accuracy of the TRI-D imager.

The imaging accuracy is very direction dependent, and the point-spread function is not a simple ellipsoid because of the sparse distribution of LOFAR stations. Some idea of imaging accuracy can be obtained from the actual distribution of sources found in the mapping procedure. From the excessive spread of the points in the radial direction (almost perpendicular to the propagation direction of the negative leader), we conclude that the imaging quality may be considerably worse in this direction, and it thus cannot be seen if the streamer activity occurs in a narrow forward cone or in a broad fanned-out structure. The propagating ionization front has a width (in the propagation direction) of less than $5 \mathrm{~m}$, which is about the locating accuracy we reach for these small intensity sources. The streamers from the flash took $100 \mu$ s to propagate over a distance of about $20 \mathrm{~m}$. The average velocity is about $2 \times 10^{5} \mathrm{~m} / \mathrm{s}$, but it can be seen that the velocity of the streamers has dropped considerably towards the end of their reach. At this distance, the following corona burst starts, as indicated by the light grey dots, some $160 \mu$ s after this burst ceases to show a VHF emission.

The structure shown in Fig. 4(b) is general for all bursts we see on the two negative leader sections we have investigated for flash B. All bursts start with some strong sources and many weaker sources, where the number decreases in time. After $100 \mu$ s this has decreased to about the background level and the next burst occurs not more than $0.5 \mathrm{~ms}$ later at the furthest distance, where streamer activity was observed from the previous flash. For the time period between the end of one burst and the start of the next, the area is VHF quiet, and we detect only background fluctuations.

The observed structure is very reminiscent of what is observed for high altitude negative leaders (HANL) in [22] using the impulsive imager. The main difference is that HANL step length covers a few $100 \mathrm{~m}$, while it is only a few $10 \mathrm{~m}$ in the present case. In the case of HANL a filament structure could be distinguished. At the end of the reach of these filaments, new corona bursts were seen to occur, a considerable time after the filament activity had died away.

A possible mechanism is that in the corona burst some poorly conductive channels (streamers) are formed that stop propagating when a maximal range is reached. Charge, however, continues to move along these streamers to accumulate at the end point. After a certain time, probably depending on conductivity and steamer length, the accumulated charge is sufficient for a breakdown creating the following corona burst. This would involve a process that may be similar to the stepping seen in laboratory when voltage changes linearly [12]. In the current standard picture for the stepping process, a space stem grows towards the head of the negative leader, where upon contact charge flows towards the outer end of the space stem, heating the space stem to become a leader, and initiating a following corona burst. In such a picture one would expect some VHF emission from the space stem when the sudden increase in (negative) charge causes a sudden drop in its potential. In this initial work we see absolutely no evidence for this process, which needs to be investigated further in future work.

The spatial development of corona bursts could not be distinguished as well on the negative leaders for flash A, although it is seen for some. This might be related to the fact that the investigated negative leaders for flash $\mathrm{A}$ are at a lower altitude than for flash B. Combined with the observations in [22], this would support a strong altitude dependence of the stepping process, as was already suggested in [23-25]. We will investigate the occurrence of corona bursts in a future, more extensive, study of negative leaders using TRI-D imaging. 


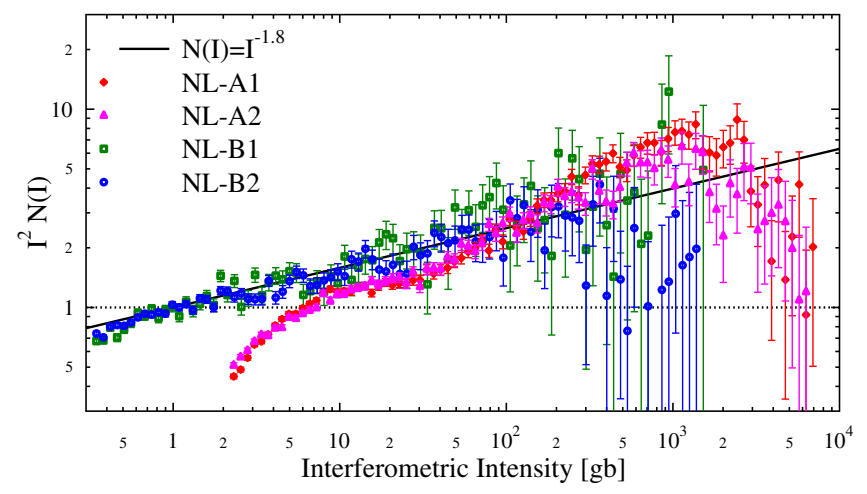

FIG. 5. Distribution of source intensities (see text for further explanation) for the four negative leaders sections given in Table I. The analysis shown is using the $\mathrm{Y}$ dipoles only. A power-law dependence is shown to guide the eye.

\section{Intensity spectrum}

The impulsive imager can reconstruct the source location of a pulse only when it is clearly distinct in the time trace. In practice this means that only for the strongest pulses the source locations can be reconstructed, and the reconstruction efficiency drops rapidly with pulse density. This imaging efficiency is difficult to quantify. Since the TRI-D imager does not rely on pulse finding, the spectrum slicing is independent of the pulse structure; the efficiency for reconstructing the source locations of the received intensity is almost $100 \%$ for sources in the image cube, with the provision that at most a single source position can be reconstructed within the time frame of a slice. In practice this means that (almost) all sources are reconstructed when the density of sources in time is less than the inverse of the slicing time. Another provision is that there should be no strong sources in the vicinity of the image cube since, otherwise, the images are blurred by the side beams from these strong sources. This completeness of the spectrum allows for an analysis of the intensity spectrum.

The distribution of the interferometric source intensities (number of sources per unit intensity and see Sec. II C for the intensity calibration) is given in Fig. 5 using a slicing time of $100 \mathrm{~ns}$, where the errors are taken as $\sqrt{N}$. The distributions are calculated for the four negative leader sections listed in Table I. The interferometry analysis is performed separately for the $\mathrm{X}$ and $\mathrm{Y}$ dipoles that have different orientations and thus different polarization sensitivities. The extracted distributions are very similar for the two dipoles, and only the results for the Y-dipole analysis is given in Fig. 5. The plotted distributions have been normalized (by dividing by a factor $F=N_{t} I_{\mathrm{Th}}$, where $N_{t}$ is the total number of sources in the distribution, and $I_{\mathrm{Th}}$ is the threshold for including sources) to express the similarity for the different leaders more clearly. Additionally, the plotted distributions have been scaled by the square of the intensity in order to emphasize smaller details. The factor $F$ is
TABLE II. The values for the normalization $\mathcal{N}$, the power $\alpha$, and the small-intensity suppression coefficient $\gamma$ in Eq. (4) as extracted for fits to the intensity distributions for the four negative leader sections listed in Table I. Separate analyses are made for the $\mathrm{X}$ and $\mathrm{Y}$ dipoles.

\begin{tabular}{lrcccc}
\hline \hline Leader & \multicolumn{1}{c}{$N_{t}$} & $I_{\mathrm{Th}}(\mathrm{gb})$ & $\mathcal{N}$ & $\alpha$ & \multicolumn{1}{c}{$\gamma$} \\
\hline NL-A1-Y & 31172 & 2. & 0.88 & 1.76 & 1.49 \\
NL-A1-X & 25454 & 2. & 0.89 & 1.79 & 1.04 \\
NL-A2-Y & 23873 & 2. & 0.85 & 1.75 & 1.24 \\
NL-A2-X & 19638 & 2. & 0.87 & 1.78 & 1.09 \\
NL-B1-Y & 9338 & .3 & 1.3 & 1.85 & 0.110 \\
NL-B1-X & 12684 & .3 & 1.0 & 1.81 & 0.135 \\
NL-B2-Y & 21207 & .3 & 1.3 & 1.88 & 0.102 \\
NL-B2-X & 29793 & .3 & 1.5 & 1.90 & 0.220 \\
\hline \hline
\end{tabular}

chosen such that a perfect inverse square power-law dependence will show as a horizontal line at unity.

The intensity distributions for the four negative leader sections are all very similar as is shown in Fig. 5. The distributions follow a power law, and to guide the eye an $I^{-1.8}$ dependence, where $\mathrm{I}$ is expressed in units of $\mathrm{gb}$, is shown by the black line. Note that because of the multiplication by $I^{2}$ the line displayed in the figure still slopes up, even though $N(I)$ is falling steeply with intensity. At smaller intensities, the distributions saturate and even drop off to zero because at most a single source, the strongest, is located per time slice of $0.1 \mu \mathrm{s}$. The weaker sources are therefore "masked" by the stronger ones, and the power spectrum is no longer complete. The main differences between the distributions show in the saturation at small intensities and is related to the measured power. The measured power depends on the altitude of the source, as well as the distance, see Sec. II B for a discussion, and differs for $\mathrm{X}$ - and $\mathrm{Y}$ dipoles. At the largest intensities there are signs of a dropoff in the power law, however, this may also be due to limited statistics.

To account for the suppression at small intensities, we fit the normalized distributions $N(I)$ with a modified power law, see Eq. (4). as follows:

$$
N(I)=\mathcal{N} I^{-\alpha} e^{-\gamma / I},
$$

where $I$ is expressed in units of gb. The last factor, dependent on $\gamma$, suppresses the distribution at small amplitudes in good agreement with the data. The values for the fitted normalization $\mathcal{N}$, the power $\alpha$, and the smallintensity suppression factor $\gamma$ are given in Table II for four negative leader sections given in Table I and the two different dipoles.

The fitted powers $\alpha$ are almost the same for all eight cases. The main differences are seen in the values for $\gamma$, indicating that for flash $\mathrm{A}$ the $\mathrm{Y}$ dipoles have a larger gain than the $\mathrm{X}$ dipoles, while for flash $\mathrm{B}$ the situation is reversed, see the discussion in Sec. III A. Since flash A occurs closer to the 
core, the $\gamma$ coefficients for flash A are all larger than those for B. In a future work we will investigate the extent of the power-law dependence in more detail, in particular, if this applies to all parts of the flash and the cutoff in the power law, which one would expect at the highest intensities since the total radiated energy should not diverge.

It is interesting to note that power-law dependencies appear in a wide range of natural and manmade phenomena, such as solar flares, earthquakes, and terrorist attacks [26-29]. Power laws often imply that there is no natural scale or size to the system since a power-law distribution is scale invariant. On the other hand, for negative leaders, it is surprising to observe a power-law distribution since one is dealing with a process where many scales enter, such as the length scale that is given by the stepping length, the time scale set by the stepping time, or the scale set by the total charge in the leader tip.

A power-law dependence has also been observed in less complete spectral data [30], using the data from the impulsive imager, where an unbiased intensity spectrum could be obtained for the $10 \%$ most intense sources.

\section{SUMMARY AND OUTLOOK}

Time resolved interferometric 3D-imaging gives an unprecedented insight in the finer dynamics of lightning propagation. Using hundreds of antennas with baselines of up to $100 \mathrm{~km}$ allows to image the more weakly emitting structures with (a better than) $10 \mathrm{~m}$ scale precision. The TRI-D imaging procedure should be considered complementary to the impulsive imager presented in [8] that builds an image by locating the sources of single, reasonably well isolated, pulses in the spectrum. The impulsive imager can efficiently image the complete flash while the TRI-Dimager allows to image much fainter aspects of the flash, as well as a somewhat improved locating accuracy, however, at the expense of being much more CPU intensive.

As a first exploratory application of TRI-D imaging, we have applied it to observe the finer details of negative leader bursts and imaged the temporal and spatial development of a corona burst. We show that it expands up to the distance where the flash of the following step starts. In addition we show that the intensities of VHF pulses emitted by negative leaders follow an almost perfect inverse-square power law extending to the largest intensities. Each of these subjects requires a much more in-depth analysis, which we plan for forthcoming publications.
In spite of the unprecedented possibilities of the present method to study the fine dynamics of lightning, there are still several aspects where the method should be improved. A minor improvement would be to optimize the antennaweighting scheme discussed in Sec. II B or implement a more sophisticated construction of the position of the maximum (or maxima), see Sec. II D. An important improvement is foreseen by fully accounting for the antenna function, which will allow for a combined analysis of the signals of the $\mathrm{X}$ and $\mathrm{Y}$ dipoles. This will remove the condition on a relatively small azimuth-angle range, see Sec. II C, but most importantly, this will give access to the direction of the electrical current moment in the VHF source and allow the implementation of a true deconvolution of the beam structure. This makes it possible to reconstruct the physical extent of the source region and, in its simplest form, to locate multiple sources per window.

The data are available from the LOFAR Long Term Archive (for access see [31]). The source code used for TRI-D imaging can be found at $[32,33]$. All figures in this work have been made using the Graphics Layout Engine [34] plotting package.

\section{ACKNOWLEDGMENTS}

The project has also received funding from the European Research Council (ERC) under the European Union's Horizon 2020 research and innovation programme Grant No. 640130. We, furthermore, acknowledge financial support from FOM [FOM-project 12PR304]; B. M. H. is supported by the NWO Grant No. VI.VENI.192.071. A. N. is supported by the DFG Grant No. NE 2031/2-1. This paper is based on data obtained with the International LOFAR Telescope (ILT). LOFAR [6] is the Low Frequency Array designed and constructed by ASTRON. It has observing, data processing, and data storage facilities in several countries that are owned by various parties (each with their own funding sources) and that are collectively operated by the ILT foundation under a joint scientific policy. The ILT resources have benefitted from the following recent major funding sources: CNRS-INSU, Observatoire de Paris, and Université d'Orléans, France; BMBF, MIWF-NRW, MPG, Germany; Science Foundation Ireland (SFI), Department of Business, Enterprise and Innovation (DBEI), Ireland; NWO, The Netherlands; The Science and Technology Facilities Council, UK. 
[1] R. Mardiana, Z.-I. Kawasaki, and T. Morimoto, J. Atmos. Sol. Terr. Phys. 64, 91 (2002).

[2] M. Akita, M. Stock, Z. Kawasaki, P. Krehbiel, W. Rison, and M. Stanley, J. Geophys. Res. 119, 6085 (2014).

[3] M. G. Stock, M. Akita, P. R. Krehbiel, W. Rison, H. E. Edens, Z. Kawasaki, and M. A. Stanley, J. Geophys. Res. 119, 3134 (2014).

[4] H. Liu, S. Qiu, and W. Dong, Atmosphere-Ocean 9, 317 (2018).

[5] D. P. Jensen, R. G. Sonnenfeld, M. A. Stanley, H. E. Edens, C. L. da Silva, and P. R. Krehbiel, J. Geophys. Res. 126, e2020JD034309 (2021).

[6] M. P. van Haarlem et al., Astron. Astrophys. 556, A2 (2013).

[7] B. M. Hare et al., Nature (London) 568, 360 (2019).

[8] O. Scholten, B. M. Hare, J. Dwyer, C. Sterpka, I. Kolmasova, O. Santolik, R. Lan, L. Uhlir, S. Buitink, A. Corstanje, H. Falcke, T. Huege, J. R. Hoerandel, G. K. Krampah, P. Mitra, K. Mulrey, A. Nelles, H. Pandya, A. Pel, J. P. Rachen et al., J. Geophys. Res. 126, e2020JD033126 (2021).

[9] M. G. Stock, Broadband interferometry of lightning, Ph.D. thesis, New Mexico Institute of Mining, 2014.

[10] J. N. Tilles, N. Liu, M. A. Stanley, P. R. Krehbiel, W. Rison, M. G. Stock, J. R. Dwyer, R. Brown, and J. Wilson, Nat. Commun. 10, 1648 (2019).

[11] K. Mulrey, A. Bonardi, S. Buitink, A. Corstanje, H. Falcke, B. Hare, J. Hoerandel, T. Huege, P. Mitra, A. Nelles, J. Rachen, L. Rossetto, P. Schellart, O. Scholten, S. ter Veen, S. Thoudam, T. Trinh, and T. Winchen, Astropart. Phys. 111, 1 (2019).

[12] P. Kochkin, A. van Deursen, and U. Ebert, J. Phys. D 47, 145203 (2014).

[13] P. O. Kochkin, A. P. J. van Deursen, and U. Ebert, J. Phys. D 48, 025205 (2015).

[14] S. Celestin, W. Xu, and V. P. Pasko, J. Geophys. Res. 120, 10712 (2015).

[15] A. Y. Kostinskiy, V. S. Syssoev, N. A. Bogatov, E. A. Mareev, M. G. Andreev, M. U. Bulatov, D. I. Sukharevsky, and V. A. Rakov, J. Geophys. Res. 123, 5360 (2018).

[16] D. S. Briggs, F. R. Schwab, and R. A. Sramek, in Synthesis Imaging in Radio Astronomy II, Astronomical Society of the Pacific Conference Series Vol. 180, edited by G. B. Taylor, C. L. Carilli, and R. A. Perley (1999), p. 127.

[17] S. Yatawatta, Mon. Not. R. Astron. Soc. 444, 790 (2014).

[18] X.-M. Shao and A. R. Jacobson, IEEE Transactions on Electromagnetic Compatibility 51, 519 (2009).

[19] B. M. Hare, O. Scholten, J. Dwyer, U. Ebert, S. Nijdam, A. Bonardi, S. Buitink, A. Corstanje, H. Falcke, T. Huege, J. R. Hörandel, G. K. Krampah, P. Mitra, K. Mulrey, B. Neijzen, A. Nelles, H. Pandya, J. P. Rachen, L. Rossetto, T. N. G. Trinh, S. ter Veen, and T. Winchen, Phys. Rev. Lett. 124, 105101 (2020).
[20] B. Neijzen, Negative leader propagation, Bachelor's thesis, University of Groningen, FSE, KVI-CART, 2019.

[21] O. Scholten et al., Sci. Rep. 11, 16256 (2021).

[22] O. Scholten et al., Atmos. Res. 260, 105688 (2021).

[23] H. E. Edens, K. B. Eack, W. Rison, and S. J. Hunyady, Geophys. Res. Lett. 41, 1336 (2014).

[24] T. Wu, S. Yoshida, Y. Akiyama, M. Stock, T. Ushio, and Z. Kawasaki, J. Geophys. Res. 120, 9071 (2015).

[25] F. Lyu, S. A. Cummer, G. Lu, X. Zhou, and J. Weinert, Geophys. Res. Lett. 43, 5516 (2016).

[26] J. A. Tenreiro Machado, C. M. A. Pinto, and A. M. Lopes, Math. Probl. Eng. 2013, 1024 (2013).

[27] F. Meng, L. N. Y. Wong, and H. Zhou, Sci. Rep. 9, 10705 (2019).

[28] M.P.H. Stumpf and M.A. Porter, Science 335, 665 (2012).

[29] Y. Virkar and A. Clauset, Ann. Appl. Stat. 8, 89 (2014).

[30] J. Machado, O. Scholten, B. Hare, S. Buitink, A. Corstanje, H. Falcke, T. Huege, J. Horandel, G. K. Krampah, P. Mitra et al., Earth Space Sci. Open Archive, 10506949 (2021).

[31] ASTRON, LOFAR Long Term Archive Access, https://www .astron.n1/lofarwiki/doku.php?id=public:lta_howto (2020). To download this data, please create an account and follow the instructions for "Staging Transient Buffer Board data" at [31]. In particular, the utility "wget" should be used as follows:

wget https://lofar-download.grid.surfsara.nl/lofigrid/SRMFifoGet.py?surl="location"where "location" should be specified as:

srm://srm.grid.sara.nl/pnfs/grid.sara.nl/data/lofar/ops/TBB/ lightning/

followed by

L668464_D20180921T194259.023Z_"stat" R000_tbo.h5 (for Flash A)

L792864_D20200814T143241.768Z_"stat" R000_tb $\bar{b} . h 5$ (for Flash B)

L792864_D20200814T143241.768Z_"stat" R000 tbo. h5 (for Flash C) and where "stat" should be replaced by the name of the station, CS001, CS002, CS003, CS004, CS005, CS006, CS007, CS011, CS013, CS017, CS021, CS024, CS026, CS028, CS030, CS031, CS032, CS101, CS103, RS106, CS201, RS205, RS208, RS210, CS301, CS302, RS305, RS306, RS307, RS310, CS401, RS406, RS407, RS409, CS501, RS503, RS508, or RS509.

[32] O. Scholten, A practical guide to lightning imaging with LOFAR, Internal Report, Kapteyn Institute, University of Groningen, NL, 2020.

[33] O. Scholten, Lofar lightning imaging, https://doi.org/ 10.5281/zenodo.4707496.

[34] C. Pugmire, S. M. Mundt, V. P. LaBella, and J. Struyf, Graphics layout engine gle 4.2.5 user manual, https://en .wikipedia.org/wiki/Graphics_Layout_Engine (2015), http:// www.gle-graphics.org/. 